\title{
Communication and Symbolic Research in Autism Spectrum Disorder: Linking Method and Theory
}

\author{
Melissa L. Allen • Charlie Lewis
}

Published online: 28 November 2014

(c) Springer Science+Business Media New York 2014

Socio-communicative difficulties remain a core diagnostic feature of Autism Spectrum Disorder (ASD), which can have a potentially severe impact on an individual's daily functioning and pose significant challenges for successfully navigating the unpredictable social world (American Psychiatric Association 2013; Klin et al. 2000). In order to communicate effectively, one must have knowledge of how symbols work, specifically that symbols refer to objects and events and can be used flexibly to represent them. The foundations of communication impairment in ASD relate to divergent pathways towards symbolic understanding, and also to differences in early interpersonal interactions. Here we present a series of 6 papers unified by a focus on symbolic and communication differences in children with ASD, a fundamental and necessary area of research required to inform our understanding and meet the needs of the growing population of individuals on the spectrum who vary in their communicative abilities.

The first paper (Lopez) pulls together diverse areas of scholarship and shows the urgent need for compatible literatures to overlap with each other, to solve the problem of how 'representations' enter the cognitive system in the first place. She does the admirable job of comparing two approaches that address parallel questions, the neuroconstructivist perspective on development and recent upsurge of analyses of communication problems associated with autism. She makes a strong case that fruitful interaction between areas of scholarship should take place if the field is to move on. She describes areas of research that are not usually in the literature. For example, the studies of how

M. L. Allen $(\bowtie) \cdot$ C. Lewis

Department of Psychology, Fylde College, Lancaster

University, Lancaster LA1 4YF, UK

e-mail: melissa.allen@lancaster.ac.uk children with ASD manipulate objects (which is part of the intersubjectivity literature) show that they do not seem to approach them in the same way-like picking a teapot up by the spout rather than the handle. This leads naturally to a consideration of how cultural practices impinge on symbol acquisition in both typically developing children and children with ASD. We include Lopez' article, in particular, because it highlights the need for research on the development of language and gesture in children with ASD to rise to Karmiloff-Smith's challenge (1998) that such work needs to be truly developmental in order to be informative or insightful. The contribution is as much a call to arms as it is an expression of how the two theoretical perspectives really fit together.

Analysis of the emerging language skills of children with ASD who have small vocabularies is notoriously hard. The second paper (Hartley and Allen) reflects meticulous approaches to data collection with minimally verbal participants who have concomitant cognitive impairment. It compares the relative influences of the 'iconicity' of a picture in linking an object to its symbolic referent. This is interesting not only because pictures allow us to compare another symbolic system with the effect of words and spoken language, but also because systems like the Picture Exchange Communication System (Bondy and Frost 1994) have been used effectively in forming a link for children with ASD between a word and the object to which it refers (although perhaps an associative one). Pictures do have a resemblance to their referents-they typically have an onomatopoeic quality. It is surprising that so little research has compared pictures and words on a foundational level given this difference between them and the use of pictures in the early education of children with a range of disabilities (see Lancioni et al. 2007). This work also shows the importance of providing data-grounded rationale for the 
types of pictures to use in augmentative systems (in this case highly iconic, color photographs), in order to build a bridge between current theory on symbolic development in ASD and educational practice.

Veneziano and Plumet perform a naturalistic analysis of pragmatic skills in children with ASD compared with matched typically developing children and those with a younger language (and chronological) age. They selected 'oppositional' exchanges because in these it is incumbent on both interlocutors to justify their position within the disagreement. Families of both typically developing children and children with ASD produced about the same number of oppositional and conflictual episodes per hour of observation, irrespective of the verbal age of the children. The fact that children with ASD are comparable to typically developing children in oppositional episodes suggests that they are exposed to as many opportunities to engage in justifications, particularly as the partners of both groups themselves engaged in the same number. The focus on oppositional episodes is interesting and echoes some of the work that Judy Dunn conducted on the emergence of sibling interactions and relationships from the late 1970s into the late 1990s (Dunn and Kendrick 1982).

The focus on siblings of children with ASD is important as this group reveals much about the intersection of biological factors and influences of the child's early language environment. By studying infants at 12 months, Talbott and TagerFlusberg allow us to understand more about whether mothers in different groups alter their input to children on the cusp of first word learning. They found that mothers of infants who were at risk of developing ASD displayed more gestures than mothers of low-risk infants, particularly in semi-structured interactions. In high-risk children these gestures correlated with the child's language 6 months later. Interestingly, these differences contrasted with a lack of a correlation between maternal and infant expressed language in the groups. Just why early links should be gestural rather than verbal is puzzling, and an area rife for additional investigation.

How far should we extend our theoretical frame when considering the nature of development of language and communicative skills? Hobson, Hobson, Cheung, and Caló consider the role of symbolic play in this framework, examining it as a medium in shared interactive engagement between language learners and their caregivers. Such links appear to relate to the child's diagnosis and the extent of the social deficit in autism. Their research promotes the importance of interpersonal engagement for the establishment of early symbolic skills in both typically developing and children with ASD. This paper has an impressive sample size and shows how the process of acquiring symbols (due to lack of social engagement) differs across populations, which then influences the development of communication and symbolic skills more generally.
The final brief report by Gomez introduces a key question in the literature on children with ASD: how much are the defining features of autism completely absent in typical development? While hand taking is NOT a defining feature of autism (and never has been) the gesture is often commented upon as a marker associated with a lack of eyecontact, which is a defining feature. Gomez' careful observations show how we need to explore the social ecology of early gestural communication in children with ASD, the value of careful observation (following Piaget) in describing and situating the complexities of early gestures and actions, and apparent overlap between children with ASD and typically developing children.

Overall, the papers in this Special Issue vary on a number of significant dimensions, but several key themes emerge. First, the heterogeneity of ASD must remain a critical focus, as individuals with different levels of ability and symptomatology present with distinct behavioural patterns and thus can be viewed as subgroups across the spectrum (Kjelgaard and Tager-Flusberg 2001; Stevens et al. 2000). Our papers include evaluation of lower-functioning, minimally verbal participants (Hartley and Allen; Gomez), siblings at high-risk for ASD (Talbott and TagerFlusberg), children with emerging (Hobson, Hobson, Cheung, and Caló) and developing (Veneziano and Plumet) verbal skills with various comparison groups (typically developing children or children with developmental disabilities). It is only when we look across these varying populations to search for commonalities and differences that we can gain a more comprehensive view of development (see Karmiloff-Smith 1998).

Second, the need for new theoretical approaches is clear. Both Lopez and Hobson and colleagues argue for a need to look beyond traditional frameworks in order to more comprehensively understand communication is ASD. In particular, Hobson et al. advocate that the study of shared interaction (measured by symbolic play) is crucial for shifts in symbolic meaning to occur. Lopez argues for a need to integrate theoretical approaches in order to benefit from a holistic perspective.

Finally, the convergence on the importance of early symbolic and communicative skills can not be overlooked, as these impact on later outcomes and developmental trajectory. We hope the papers in this volume will spark further debate about the appropriate methods for studying the links between symbolic and communicative skills in ASD and more importantly, their underpinning theoretical foundations.

\section{References}

American Psychiatric Association. (2013). Diagnostic and statistical manual of mental disorders (5th ed.). Washington, DC: Author. 
Bondy, A. S., \& Frost, L. A. (1994). The picture exchange communication system. Focus on Autism and Other Developmental Disabilities, 9(3), 1-19.

Dunn, J., \& Kendrick, C. (1982). Siblings: Love, envy, and understanding (pp. 24-57). Cambridge, MA: Harvard University Press.

Karmiloff-Smith, A. (1998). Development itself is the key to understanding developmental disorders. Trends in Cognitive Sciences, 2(10), 389-398.

Kjelgaard, M. M., \& Tager-Flusberg, H. (2001). An investigation of language impairment in autism: Implications for genetic subgroups. Language and Cognitive Processes, 16(2-3), 287-308.

Klin, A., Schultz, R., \& Cohen, D. (2000). Theory of mind in action: developmental perspectives on social neuroscience. In S. Baron-
Cohen, H. Tager-Flusberg, \& D. Cohen (Eds.), Understanding other minds: Perspectives from developmental neuroscience (2nd ed., pp. 357-388). Oxford: Oxford University Press.

Lancioni, G. E., O'Reilly, M. F., Cuvo, A. J., Singh, N. N., Sigafoos, J., \& Didden, R. (2007). PECS and VOCAs to enable students with developmental disabilities to make requests: An overview of the literature. Research in Developmental Disabilities, 28(5), 468-488.

Stevens, M. C., Fein, D. A., Dunn, M., Allen, D., Waterhouse, L. H., Feinstein, C., \& Rapin, I. (2000). Subgroups of children with autism by cluster analysis: A longitudinal examination. Journal of the American Academy of Child and Adolescent Psychiatry, 39(3), 346-352. 